\section{Distribuição espacial dos idosos de um município de médio porte do interior paulista segundo algumas características sócio-demográficas e de morbidade}

\author{
Spatial distribution of elderly individuals in a \\ medium-sized city in São Paulo State, Brazil, \\ according to key socio-demographic and morbidity \\ characteristics
}

\begin{abstract}
Geographic Information Systems serve as important public health tools for analyzing population disease distribution and thus for identifying individuals with chronic non-communicable diseases. The current study performed a spatial analysis of the distribution of the population 60 years and older in the city of Botucatu, São Paulo State, Brazil, studying the socio-demographic profile and the presence of diabetes mellitus and arterial hypertension. Data were analyzed from 468 elderly individuals from a population survey conducted in 2001-2002. Elders with the highest socioeconomic status live in the census tracts with the highest social strata, as proven statistically by spatial analysis techniques for income and schooling. No spatial distribution pattern was found for elderly individuals with hypertension and diabetes, who were located heterogeneously on the map. The study suggests the use of geoprocessing techniques for digital mapping of areas covered by primary health care units, aimed at better monitoring of the distribution of elderly individuals with chronic diseases and their care by health professionals.
\end{abstract}

Geographic Information Systems; Residence Characteristics; Aged; Primary Health Care
Frederico Grizzi de Campos ${ }^{1}$

Ligia Vizeu Barrozo 2

Tania Ruiz ${ }^{3}$

Chester Luiz Galvão César 4

Marilisa Berti Azevedo Barros 5

Luana Carandina 3

Moisés Goldbaum 6

\section{Introdução}

Os idosos representam grande parcela da demanda dos serviços de saúde em qualquer nível de hierarquia do sistema 1,2. Diversas patologias crônicas não transmissíveis, como hipertensão arterial, diabetes mellitus, câncer, osteoartrites, entre outras, têm maior prevalência em faixas etárias mais altas 3,4 , sendo, portanto, cada vez mais encontradas na população, e gerando, dessa forma, maior exigência dos serviços de saúde na atenção a esses pacientes 5,6. A tendência atual de retangularização da pirâmide demográfica brasileira cada vez mais requer estudos sobre a população idosa 7 .

Os serviços de saúde podem atuar de forma a alterar a freqüência, a distribuição dos agravos à saúde e até mesmo a desigualdade entre setores sociais, melhorando a qualidade de vida da população. Por tal razão, o conhecimento do perfil dos agravos à saúde prevalentes em uma região é um indicador insubstituível para a provisão de serviços e de recursos usados em atividades de natureza preventiva, diagnóstica, terapêutica e de reabilitação ${ }^{8}$.

O Programa Agentes Comunitários de Saúde (PACS), implantado em 1991, e o Programa Saúde da Família (PSF), implantado a partir de 1994, representaram uma evolução no atendimento do Sistema Único de Saúde (SUS) no nível da atenção básica, promovendo uma maior interação com a comunidade, por meio dos agentes comu- 
nitários de saúde, e, essencialmente, promovendo ações voltadas à prevenção de agravos à saúde nas diversas faixas etárias, desde antes do nascimento até a terceira idade 9 . O PACS incorporou o conceito de territorialização, essencial para o planejamento na implantação do PSF. Na gestão de uma unidade de saúde, a área de influência permeia a área coberta pela equipe de saúde de uma unidade, e sua abrangência corresponde à área geográfica de sua responsabilidade, onde se desenvolve o processo de apropriação do espaço local 10. Os índices de prevalência de agravos à saúde numa população adscrita também são levados em conta na territorialização para a instalação de uma unidade de saúde, refletindo a importância do estudo da distribuição espacial das patologias 10 .

Green 11 estudou a prevalência de diabetes mellitus na área urbana de Winnipeg, Canadá, demonstrando que taxas altas da doença se agrupavam significativamente no espaço devido às características étnicas, sócio-econômicas, ambientais e de estilo de vida da população. Freqüentemente, recorre-se às técnicas cartográficas para mapeamento das taxas da doença, na tentativa de identificação das áreas com altas taxas, já que os mapas podem sugerir hipóteses sobre a etiologia. No entanto, apenas identificar visualmente a presença de altas taxas pode levar a conclusões incorretas. Existem questões importantes que devem ser consideradas na análise de um mapa. A primeira observação diz respeito ao ponto de corte adotado para a definição dos intervalos das classes em mapas coropléticos. Diferentes pontos de corte levam a diferentes interpretações e padrões espaciais 12 . Além disso, deve-se considerar também que a percepção humana tem a tendência de encontrar padrões até mesmo onde eles não existem de fato. Outros aspectos importantes dizem respeito à escala adotada, à "falácia ecológica", à flutuação dos dados em eventos raros e ao "problema da unidade de área modificável”, onde os resultados com base em dados agregados dependem da unidade de agregação ${ }^{13}$, entre outros. Dessa forma, a análise estatística espacial serve como poderoso instrumento para verificação do padrão espacial das patologias e variáveis sócio-econômicas.

A cidade de Botucatu, localizada no interior paulista brasileiro a cerca de 240km de São Paulo, capital, tem uma população aproximada de 118 mil habitantes (Instituto Brasileiro de Geografia e Estatística. Projeção da população do Brasil: 19802050. http://www.ibge.gov.br/home/estatistica/ populacao/projecao_da_populacao/, acessado em 08/Set/2005). O PSF foi implantado recentemente na cidade, tendo, como início de suas atividades, o segundo semestre do ano de 2003
(Prefeitura Municipal de Botucatu. http://www. botucatu.sp.gov.br, acessado em 15/Jun/2005). O presente estudo analisou a distribuição espacial dos idosos segundo características demográficas, sociais e de morbidade mais prevalente (hipertensão arterial e diabetes mellitus), visando identificar possíveis diferenciais intra-urbanos para a melhor compreensão da distribuição dessas patologias no município e permitir o melhor planejamento dos serviços de saúde.

\section{Materiais e métodos}

\section{Banco de dados}

Entre os anos de 2001 e 2002, foi realizado um estudo multicêntrico abrangendo as cidades de Botucatu, Campinas e zona oeste da cidade de São Paulo. O Inquérito Multicêntrico de Saúde no Estado de São Paulo (ISA-SP) e ISA-Capital teve como objetivo diagnosticar a morbidade referida na população, as condições de saúde e a utilização de serviços, através de entrevista domiciliar 14. Nesse inquérito, as informações foram referenciadas territorialmente.

O estudo desenvolvido foi de base populacional, correspondendo à população não institucionalizada, residente na área urbana da cidade de Botucatu. Para o planejamento de campo, foram extraídas informações do banco de dados do Instituto Brasileiro de Geografia e Estatística (IBGE), referentes à contagem da população de 1996. Utilizou-se amostragem por conglomerados em dois estágios para sorteio, respectivamente, dos setores censitários e dos domicílios, incluindose, neste estudo, apenas os sujeitos de ambos os sexos, com 60 anos ou mais. A amostra totalizou 468 pessoas, com variação entre 60 e 97 anos no momento da coleta dos dados, de um total de 1.588 pessoas entrevistadas 14 .

Em Botucatu, a planta cadastral, digitalizada pela Secretaria de Planejamento do Município, foi tomada como base cartográfica e incorporada a um sistema de informações geográficas. Os endereços dos idosos entrevistados foram georeferenciados na base de dados espacial. Em 1996, o Município de Botucatu compreendia 95 setores censitários, segundo a definição do IBGE. De acordo com o delineamento amostral do inquérito, foram sorteados trinta setores. Os centróides dos polígonos formados pelos 95 setores foram calculados, e, com suas coordenadas geográficas, foi possível selecionar apenas as referentes aos trinta sorteados. Com as coordenadas desses trinta setores, foram traçados novos polígonos, pela poligonização de Thiessen, abrangendo todo o perímetro urbano de 
Botucatu. Esse novo mapa foi utilizado para as análises espaciais.

As distribuições espaciais das seguintes variáveis foram estudadas: sexo, faixas etárias, escolaridade do chefe da família, faixa de renda individual, referência de hipertensão arterial e referência de diabetes mellitus pelo entrevistado.

\section{Análise espacial}

Realizou-se uma análise exploratória dos dados espaciais utilizando-se o programa GeoDa v0.95 (Spatial Analysis Laboratory, University of Illinois at Urbana-Champaign, Urbana, Estados Unidos) 15 . Tal análise objetivou descrever a distribuição das variáveis do estudo, identificar observações atípicas (outliers) não só em relação ao tipo de distribuição, mas também em relação aos vizinhos, e buscar a existência de padrões na distribuição espacial dos eventos.

Inicialmente, foram elaborados mapas do percentual de cada variável utilizando-se, como ponto de corte para o intervalo das classes, unidades de desvio-padrão. Para o estudo do padrão espacial dos idosos segundo suas condições socioeconômicas, foram escolhidas as variáveis escolaridade e renda. Foram mapeados o percentual de idosos com escolaridade de até primeiro grau completo e o percentual de idosos com renda superior a dez salários mínimos. Essas duas variáveis permitiram avaliar se existe desigualdade social na ocupação do espaço na cidade de Botucatu. Foi também mapeado o percentual de idosos com hipertensão e diabetes mellitus autoreferidas.

Para verificar se as variáveis apresentaram autocorrelação espacial global, foram calculados os coeficientes I de Moran para as variáveis socioeconômicas e para as patologias auto-referidas, com matrizes de pesos de contigüidade binária, segundo convenção Rook. Como os eventos eram referentes à proporção, foram também aplicados os testes de Moran com estimativa Bayesiana empírica para taxas para as patologias. Essa abordagem procura minimizar o problema de instabilidade das taxas brutas na estimação de risco em pequenas áreas 15,16,17. Como o número de pessoas estudadas foi pequeno, qualquer leve alteração no número de eventos, mesmo que devido ao mero acaso, provocariam grandes variações no valor da taxa. Marshall 16 propôs a aplicação de estimadores Bayesianos empíricos como alternativa ao uso de taxas brutas nessas condições. Essa estimativa calcula a média ponderada entre a taxa bruta para cada setor e a média de uma janela espacial, com pesos proporcionais à população em risco.
Também foram calculados indicadores de associação local. O indicador Moran local foi aplicado para identificar possíveis focos de localizações atípicas. Os coeficientes de autocorrelação espacial global e associação local foram considerados significativos quando $\mathrm{p}<0,05$. Os valores de probabilidade fornecidos (valores de p) devem ser vistos como níveis de pseudo-significância, tendo em vista que as inferências são feitas a partir da abordagem das permutações.

\section{Resultados}

\section{Características sócio-demográficas dos idosos de Botucatu}

Dos 468 idosos entrevistados no Município de Botucatu, $54 \%$ eram do sexo feminino, e $46 \%$, do masculino. Cerca de $52 \%$ dos idosos entrevistados encontravam-se na faixa etária entre 60 e 70 anos, $33 \%$ entre 71 e 80 anos, e 15\% acima dos 81 anos.

Praticamente, $20 \%$ dos idosos entrevistados não freqüentaram escola, e cerca de $50 \%$ possuíam apenas até o lo grau completo. Em torno de $42,5 \%$ recebiam rendimentos de até três salários mínimos, em geral, provenientes de aposentadoria e pensões. A distribuição espacial do percentual de idosos com escolaridade até lo grau completo (Figura 1) sugere possível autocorrelação espacial para essa variável. Observa-se que o eixo centro-oeste e dois setores ao norte apresentam os melhores indicadores para escolaridade, com percentuais abaixo da média.

A desigualdade social entre os idosos do município apresentou-se ainda mais nítida para a variável renda (Figura 2). O eixo centro-oeste e um distrito ao sul apresentaram proporção acima da média para idosos com renda acima de dez salários mínimos. Pode-se dizer que o padrão espacial das variáveis sócio-econômicas em Botucatu segue o da maioria das cidades brasileiras, onde o arranjo espacial concentra áreas mais valorizadas na porção central da cidade. O eixo de nível sócio-econômico mais alto no sentido oeste, em Botucatu, explica-se em função dos bairros nobres formados em direção ao campus da Universidade Estadual Paulista (UNESP) e a um condomínio de alto padrão à margem da Rodovia Marechal Rondon.

A autocorrelação espacial global sugerida pelos mapas das Figuras 1 e 2 foi confirmada pelo coeficiente I de Moran para as duas variáveis (Tabela 1). Além dessa tendência global, as variáveis sócio-econômicas também apresentaram agrupamentos locais. Alguns polígonos no centro da cidade formaram um conglomerado 
Distribuição espacial do percentual de idosos, chefes de família, com escolaridade de até 1o grau completo, por setor amostrado. Botucatu, São Paulo, Brasil, 2001-2002.

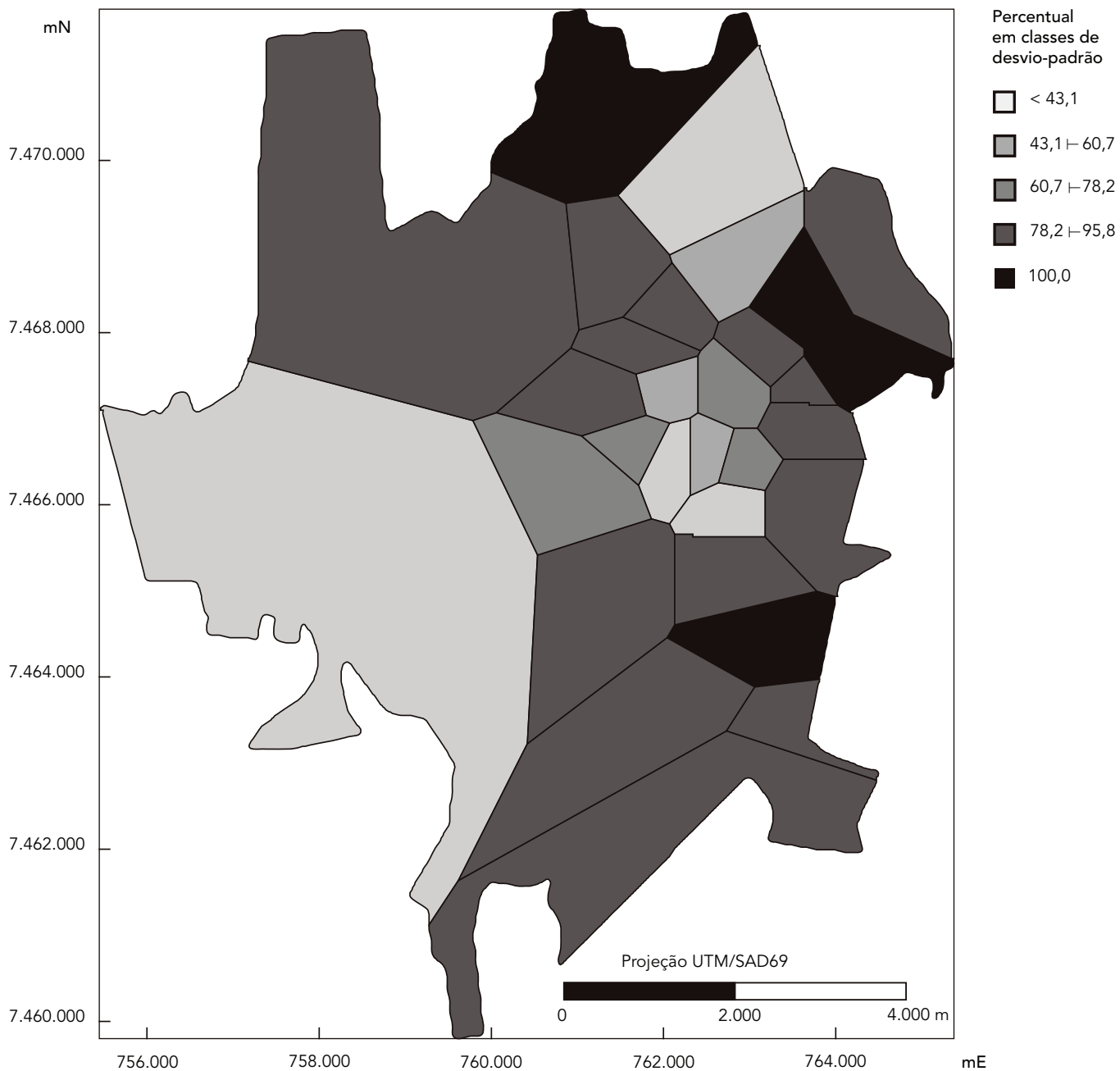

significativo com menor percentual de idosos com escolaridade de até 1o grau completo, quase coincidente com o conglomerado com alto percentual de idosos com renda acima de dez salários mínimos.

\section{Hipertensão e diabetes mellitus nos idosos de Botucatu}

Cerca de $94 \%$ dos entrevistados tiveram sua pressão arterial aferida há menos de um ano. Esse intervalo é considerado como de boa periodicidade para o diagnóstico de hipertensão 18. A Tabela 2 mostra que a maior prevalência de hipertensão auto-referida ocorre entre as mulheres (52,6\%), enquanto apenas $34,5 \%$ dos homens se declararam portadores da patologia.

$\mathrm{O}$ diabetes mellitus foi auto-referido em $13,1 \%$ da população entrevistada (Tabela 2), sendo mais prevalente no sexo feminino (15\%).

Os padrões espaciais das patologias autoreferidas estudadas podem ser observados nas Figuras 3 e 4 . Os mapas não apresentaram organização espacial definida para ambas as patologias. O padrão aleatório foi verificado para diabete mellitus, que apresentou valor de $\mathrm{p}$ não significativo para coeficiente I de Moran de 0,04 ( $p=0,23)$, mesmo para o teste de Moran com 
Distribuição espacial do percentual de idosos, chefes de família, com renda superior a dez salários mínimos, por setor amostrado. Botucatu, São Paulo, Brasil, 2001-2002.

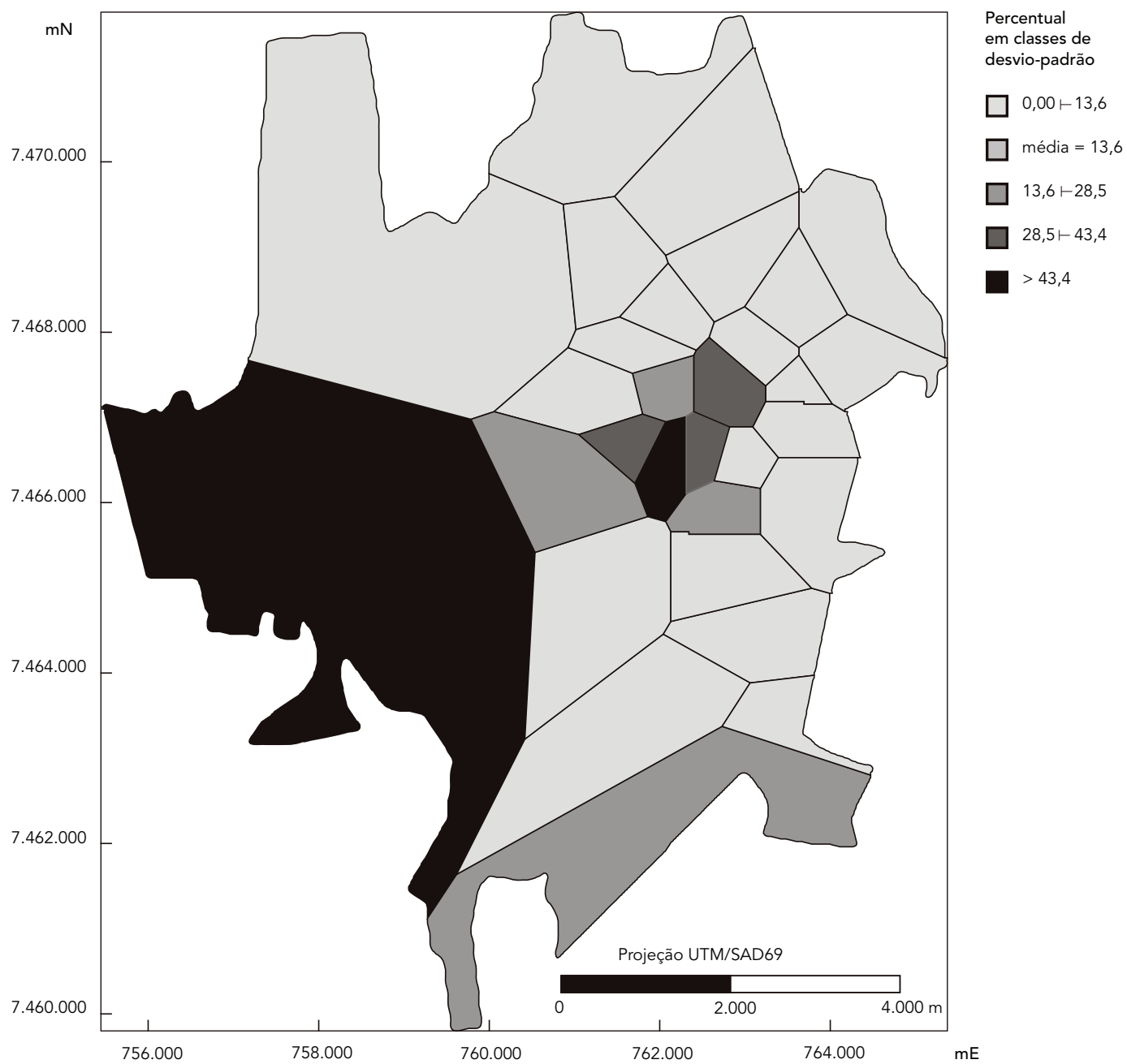

estimativa Bayesiana empírica para taxas (coeficiente I de Moran = -0,15; p = 0,08) (Tabela 1). A hipertensão arterial apresentou I de Moran negativo, com valores de $\mathrm{p}$ significativos em ambos os testes.

Embora não tenha sido observada dependência espacial global positiva para hipertensão arterial ou diabetes (demonstrado pelos valores negativos na tabela de autocorrelação espacial), alguns polígonos poderiam apresentar valores locais discrepantes. No entanto, os testes para Moran local não identificaram conglomerados significativos dessas patologias.

\section{Discussão}

A população de idosos do Município de Botucatu $(11,2 \%)$ é superior à da média nacional $(8,6 \%)$ e à da média do Estado de São Paulo (9\%), aproximando-se da taxa encontrada para o Rio Grande do Sul (10,5\%) (IBGE. Censo demográfico 2000. http://www.ibge.gov.br).

Uma das limitações a ser lembrada para o presente estudo é o fato de que os dados utilizados são produto de informações de um inquérito de base populacional, cujo desenho foi elaborado para obter amostra apropriada para análises 
Indicador global de autocorrelação espacial para proporção de idosos chefes de família com escolaridade de até 1o grau completo, proporção de idosos com renda superior a dez salários mínimos, hipertensão arterial e diabetes mellitus. Botucatu, São Paulo, Brasil, $2001-2002$.

\begin{tabular}{|c|c|c|c|c|}
\hline Variável & $\begin{array}{l}\text { Coeficiente I } \\
\text { de Moran }\end{array}$ & Valor de $p$ * & $\begin{array}{c}\text { Coeficiente I de Moran } \\
\text { (estimativa bayesiana empírica) }\end{array}$ & Valor de $p$ * \\
\hline Escolaridade até 1o grau & 0,23 & 0,015 & ** & ** \\
\hline Renda superior a dez salários mínimos & 0,25 & 0,010 & ** & ** \\
\hline Hipertensão arterial & $-0,21$ & 0,020 & $-0,24$ & 0,001 \\
\hline Diabetes mellitus & 0,04 & 0,230 & $-0,15$ & 0,080 \\
\hline
\end{tabular}

* Pseudo-significância empírica com base em 10 mil permutações;

** Não calculado.

Tabela 2

Prevalência auto-referida de hipertensão arterial e diabetes mellitus na população de 60 anos e mais, por sexo. Botucatu, São Paulo, Brasil.

\begin{tabular}{|c|c|c|c|c|c|c|c|c|}
\hline \multirow[t]{3}{*}{ Sexo } & \multicolumn{8}{|c|}{ Variáveis } \\
\hline & \multicolumn{4}{|c|}{ Hipertensão arterial } & \multicolumn{4}{|c|}{ Diabetes mellitus } \\
\hline & Não (\%) & $\operatorname{Sim}(\%)$ & Não sabe (\%) & Total & Não (\%) & $\operatorname{Sim}(\%)$ & Não sabe (\%) & Total \\
\hline Masculino & $139(64,6)$ & $74(34,5)$ & $2(0,9)$ & 215 & $192(89,3)$ & $23(10,7)$ & $0(0,0)$ & 215 \\
\hline Feminino & $120(47,4)$ & $133(52,6)$ & $0(0,0)$ & 253 & $213(84,2)$ & $38(15,0)$ & $2(0,8)$ & 253 \\
\hline Total & $259(55,4)$ & $207(44,2)$ & $2(0,4)$ & 468 & $405(86,5)$ & $61(13,1)$ & $2(0,4)$ & 468 \\
\hline
\end{tabular}

de base individual relativos à população do município como um todo (detalhes da metodologia do plano de amostragem podem ser encontrados em Cesar et al. ${ }^{19}$ ). A amostra sorteada tem poder suficiente e "representatividade" adequada apenas para análises da população total do município e não para a população de bairros ou de setores censitários. Apesar disto, foi possível detectar a presença de padrão espacial em relação aos dois indicadores de nível sócio-econômico pelas análises espaciais desenvolvidas.

A baixa escolaridade dos idosos reflete a desigualdade social e as políticas de educação predominantes nas décadas de 1930 e 1940, quando o acesso à escola ainda era muito restrito. Considerando o número médio de anos de estudo dos idosos responsáveis pelos domicílios no Brasil, o valor encontrado, no ano de 2000, pelo Censo Demográfico, era de apenas 3,4 anos (3,5 anos para os homens e 3,1 anos para as mulheres). No Município de Botucatu, tal desigualdade pode ser verificada também no espaço. Os dados individuais coletados, quando analisados de forma agregada por polígono, revelaram um arranjo espacial não aleatório, em que os idosos com melhor escolaridade e renda residiam na por- ção central do perímetro urbano e nos bairros residenciais de padrão mais elevado, a oeste. $\mathrm{Na}$ periferia, residiam os idosos de nível sócio-econômico mais baixo. A autocorrelação espacial positiva encontrada para renda e escolaridade sugeriu que essas duas variáveis são sensíveis, entre os idosos de Botucatu, para detectar os indivíduos de maior risco social e que devem ser mais assistidos pelas equipes de saúde da família do município.

Segundo os índices do Ministério da Saúde 20, a prevalência de diabetes mellitus aumenta com a faixa etária, atingindo $17 \%$ dos maiores de sessenta anos, enquanto a prevalência de hipertensão em idosos atinge $65 \% 21$. Tem sido reportado que tanto o diabetes quanto a hipertensão são mais prevalentes no sexo masculino. $\mathrm{O}$ inquérito de Botucatu mostrou que $13,1 \%$ dos entrevistados se referiram como portadores de diabetes, $\mathrm{e}$ $44,2 \%$, de hipertensão arterial, com maior prevalência para o sexo feminino.

A análise espacial dos resultados do inquérito de Botucatu não identificou agrupamentos espaciais para hipertensão arterial e diabetes mellitus. Pode-se pensar que a população idosa de Botucatu apresenta homogeneidade do 
Distribuição espacial do percentual de idosos com hipertensão arterial auto-referida no inquérito. Botucatu, São Paulo, Brasil, 2001-2002.

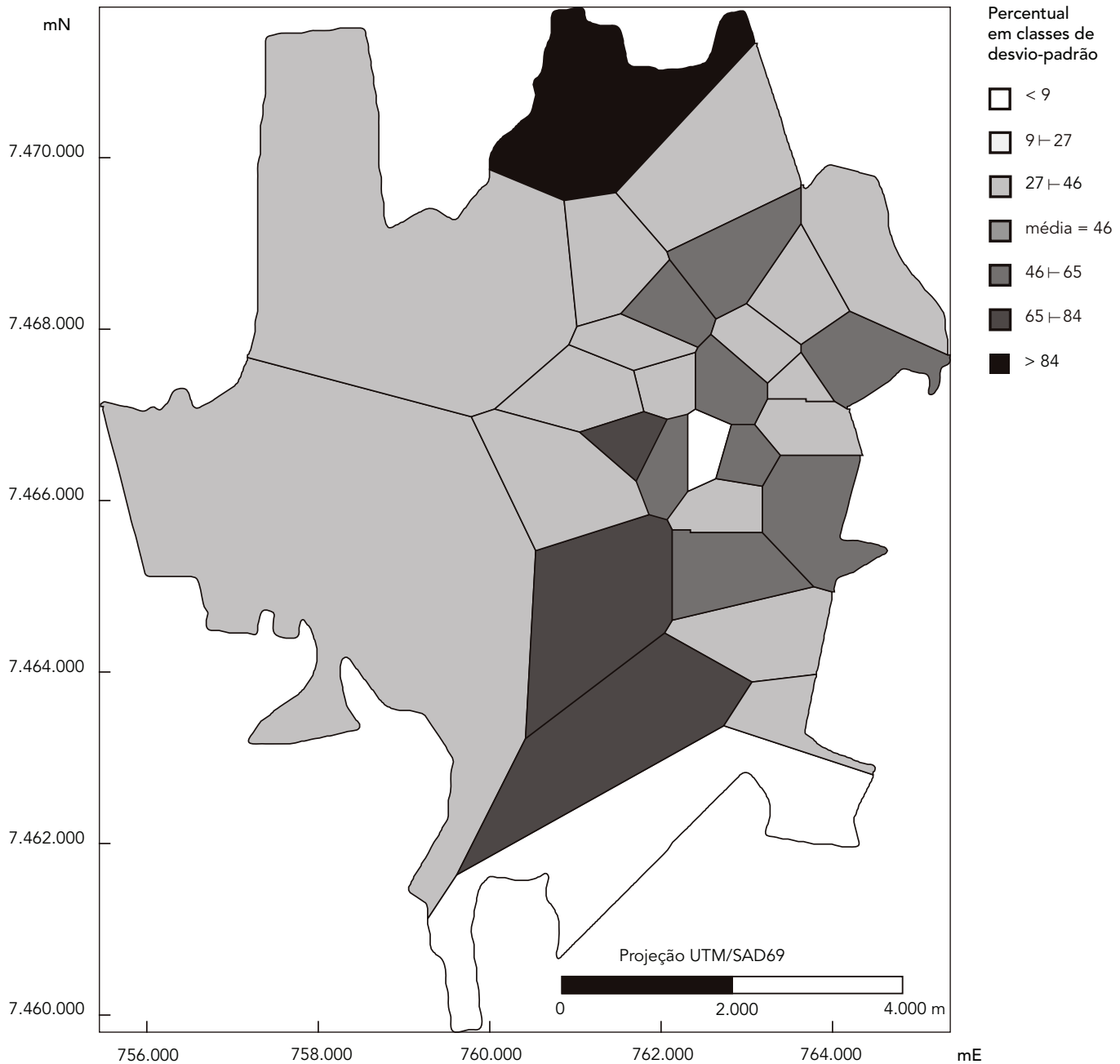

ponto de vista étnico e de estilo de vida, o que seria bastante plausível. A observação de homogeneidade espacial quanto à prevalência de hipertensão, ao mesmo tempo em que foram observadas diferenças espaciais quanto ao nível sócio-econômico, contraria o observado em vários estudos. Zaitune et al. 21, analisando a hipertensão em idosos de Campinas, São Paulo, utilizando dados do ISA-SP, verificaram associação significativa, que persistiu no modelo final, entre o nível de escolaridade e a prevalência de hipertensão. Cesar et al. 19, com os dados globais do ISA-SP, verificaram, em pessoas com vinte anos ou mais de idade, prevalência de hipertensão de $26,4 \%$ no segmento social cujo chefe da família tinha menos de quatro anos de escolari- dade e de $15,1 \%$ entre aqueles de famílias cujos chefes tinham 12 ou mais anos de escolaridade. Com base nos dados da Pesquisa Nacional por Amostra de Domicílios de 2003 (PNAD 2003), Barros et al. 22 verificaram, em pessoas com 18 anos ou mais de idade, razão de prevalência de 2,9 (ajustada para sexo, idade, macrorregião de residência e tipo de respondente) nas pessoas com menos de quatro anos de escolaridade em relação ao segmento com 11 anos ou mais. Resultados semelhantes foram observados na pesquisa realizada pelo Instituto Nacional de Câncer (INCA) 23 e em estudos realizados em outros países 24,25,26.

O Programa de Atenção à Saúde do Adulto, dentro do PSF, tem como base a prevenção e cui- 
Distribuição espacial do percentual de idosos com diabetes mellitus auto-referida no inquérito. Botucatu, São Paulo, Brasil, 2001-2002.

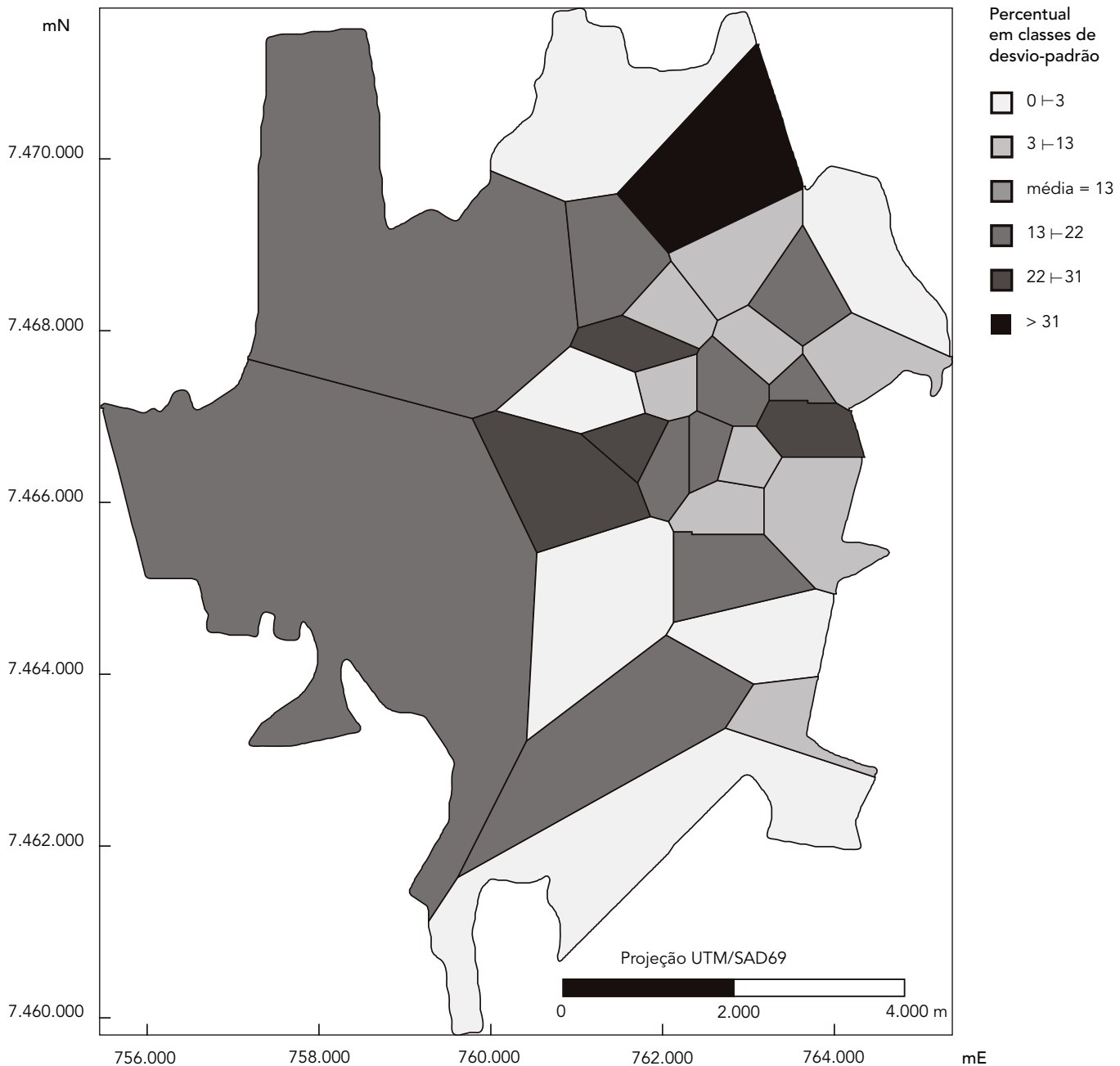

dados de pacientes portadores de hipertensão e diabetes, doenças crônicas cuja prevalência as coloca como as mais freqüentes e responsáveis por grande índice de morbi-mortalidade, na faixa etária em questão ${ }^{27}$. As análises espaciais, quando utilizadas ao nível local de atenção à saúde, podem promover o desenvolvimento de um programa de atenção à saúde do adulto mais eficiente, pois, pelo mapeamento e localização dos idosos de risco, pode ser traçado um perfil de visitas domiciliares a serem realizadas pela equipe de saúde, desde os agentes comunitários de saúde até os médicos de família. Esses instrumentos são ainda de maior utilidade em patologias de características infecto-contagiosas para localizá-las segundo associação com a distribuição espacial e traçar planos de isolamento e metas para combater os agentes infecciosos ou transmissores. Além disso, o geoprocessamento pode ser utilizado como técnica para orientar as administrações municipais no planejamento da distribuição de novas unidades de saúde da família no território do município, priorizando as áreas que apresentem piores índices sócio-econômicos. Por meio da análise espacial das características de renda e escolaridade dos idosos do Município de Botucatu, notou-se que as unidades de saúde da família foram estrategicamente 
planejadas nos locais onde a população possui um nível social mais baixo.

\section{Conclusões}

A análise de distribuição espacial da prevalência de hipertensão arterial e diabetes em uma população de idosos de Botucatu demonstrou não haver um padrão espacial global ou local entre as duas doenças analisadas, o que provavelmente se explique pela homogeneidade da população idosa nesse município, no que se refere aos padrões étnicos e ao estilo de vida, aliado ao subdiagnóstico. Entretanto, mostrou eficácia para apontar as áreas da cidade com idosos de risco social.

Os instrumentos de análise geoespacial parecem ser importantes recursos que podem ser mais explorados e utilizados em saúde pública e no PSF, pois permitem localizar usuários de risco, promovendo um melhor planejamento na assistência a esses indivíduos pela equipe de saúde, e, conseqüentemente, dando uma melhor qualidade de vida à população assistida. Sugere-se a utilização rotineira da tecnologia do geoprocessamento nas unidades de saúde da família, para um melhor planejamento da abordagem local da população e do trabalho de visitas domiciliares de toda equipe de saúde.

\section{Resumo}

Os Sistemas de Informação Geográfica constituem importantes instrumentos dentro da Saúde Pública como técnicas de análise da distribuição de agravos à população, e, portanto, podem ser usados no estudo da localização de indivíduos portadores de doenças crônicas. Este trabalho objetivou a realização de análise espacial da distribuição da população de sessenta anos e mais no Município de Botucatu, São Paulo, Brasil, estudando o perfil sócio-demográfico e a presença de diabetes e hipertensão arterial. Foram analisados dados de 468 idosos da amostra de inquérito populacional realizado entre 2001 e 2002. Os idosos com melhor nível sócio-econômico residem nos setores censitários de estratos sociais mais altos, o que foi estatisticamente comprovado pela utilização de técnicas de análise espacial para renda e escolaridade. Não se encontrou padrão de distribuição espacial para idosos hipertensos e diabéticos que se localizaram no mapa de forma heterogênea. O presente estudo sugere a utilização das técnicas de geoprocessamento para o mapeamento digital das áreas de abrangências das Unidades de Atenção Primária à Saúde, para um melhor controle da distribuição de idosos portadores de doenças crônicas e de sua assistência pelos profissionais de saúde.

Sistemas de Informação Geográfica; Distribuição Espacial da População; Idoso; Atenção Básica à Saúde

\section{Colaboradores}

F. G. Campos idealizou o tema, fez pesquisa bibliográfica e análise dos dados levantados. L. V. Barrozo colaborou na análise de geoprocessamento dos dados encontrados e confecção dos mapas de distribuição populacional. T. Ruiz confeccionou o resumo deste artigo. C. L. G. César, M. B. A. Barros, L. Carandina e M. Goldbaum cederam os dados populacionais para análise e confecção deste artigo, avaliaram e criticaram o artigo e fizeram várias sugestões que foram agregadas no texto original. 


\section{Referências}

1. Lima-Costa MFF, Guerra HL, Firmo JOA,Vidigal PG, Uchoa E, Barreto SM. The Bambuí Health and Aging Study (BHAS): private health plan and medical care utilization by older adults. Cad Saúde Pública 2002; 18:177-86.

2. Pacheco Ríos A, Herrera Basto E, Sánchez Ahedo R, Santibañez Freg MP, Martínez Plasencia X, Fernández Ortega MA. Utilización de los servicios de salud por población anciana. Rev Fac Med UNAM 2001; 44:239-43.

3. Firmo JOA, Uchoa E, Lima-Costa MF. Projeto Bambuí: fatores associados ao conhecimento da condição de hipertenso entre idosos. Cad Saúde Pública 2004; 20:512-21.

4. Taddei CFG, Ramos LR, Moraes JC, Wajngarten M, Libberman A, Santos SC, et al. Estudo multicêntrico de idosos atendidos em ambulatórios de cardiologia e geriatria de instituições brasileiras. Arq Bras Cardiol 1997; 69:327-33

5. Xavier FMF, Ferraz MPT, Marc NA. Definição dos idosos de qualidade de vida. Rev Bras Psiquiatr 2003; 25:31-9.

6. Santos SR, Santos IBC, Fernandes MGM. Qualidade de vida do idoso na comunidade: aplicação da escala de Flanagan. Rev Latinoam Enferm 2002; 10:757-64.

7. Chaimowicz F. A saúde dos idosos brasileiros às vésperas do século XXI: problemas, projeções e alternativas. Rev Saúde Pública 1997, 31:184-200.

8. Pereira MG. Serviços de saúde. In: Pereira MG, organizador. Epidemiologia: teoria e prática. Rio de Janeiro: Editora Guanabara Koogan; 1995. p. 513-37.

9. Silvestre JA, Costa Neto MM. Abordagem do idoso em programas de saúde da família. Cad Saúde Pública 2003; 19:839-47.

10. Unglert CVS. Territorialização em saúde: a conquista do espaço local enquanto prática do planejamento ascendente [Tese de Livre Docência]. São Paulo: Faculdade de Saúde Pública, Universidade de São Paulo; 1995.

11. Green C, Hoppa RD, Young TK, Blanchard JF. Geographic analysis of diabetes prevalence in an urban area. Soc Sci Med 2003; 57:551-60.

12. Bartels CJ, Beurden AUCJ. Using geographic and cartographic principles for environmental assessment and risk mapping. J Hazard Mater 1998; 61:115-24.

13. Waller LA. A civil action and statistical assessments of the spatial pattern of disease: do we have a cluster? Regul Toxicol Pharmacol 2000; 32:174-83.

14. César CLG, Goldbaum M, Barros MBA, Carandina L. Condições de vida e saúde de populações: Inquérito Multicêntrico de Saúde no Estado de São Paulo: ISA-SP e ISA-Capital. São Paulo: Faculdade de Saúde Pública, Universidade de São Paulo; 2002.
15. Anselin L, Syabri I, Kho Y. GeoDa: an introduction to spatial data analysis. Geogr Anal 2005; 38:5-22.

16. Marshall RJ. Mapping disease and mortality rates using empirical Bayes estimators. J R Stat Soc Ser C Appl Stat 1991; 40:283-94.

17. Assunção RM, Reis EA. A new proposal to adjust Moran's I for population density. Stat Med 1999; 18:2147-62.

18. Mion Jr. D, Machado CA, Gomes MAM, Nobre F, Kohlmann Jr. O, Amodeo C, et al. IV diretrizes brasileiras de hipertensão arterial. Arq Bras Cardiol 2004; 82 Suppl 4:1-1.

19. Cesar CLG, Carandina L, Alves MCP, Barros MBA, Goldbaum M. Saúde e condição de vida em São Paulo: inquérito multicêntrico de saúde no Estado de São Paulo - ISA/SP. São Paulo: Faculdade de Saúde Pública, Universidade de São Paulo; 2005.

20. Departamento de Informática do SUS. Taxas de prevalência de diabetes mellitus, 1988. http:// tabnet.datasus.gov.br/cgi/idb1998/fqd09.htm (acessado em 05/Out/2005).

21. Zaitune MPA, Barros MBA, César CLG, Carandina L, Goldbaum M. Hipertensão arterial em idosos: prevalência, fatores associados e práticas de controle no Município de Campinas, São Paulo, Brasil. Cad Saúde Pública 2006; 22:285-94.

22. Barros MBA, César CLG, Carandina L, Torre GD. Desigualdades sociais na prevalência de doenças crônicas no Brasil, PNAD-2003. Ciênc Saúde Coletiva 2006; 11:911-26.

23. Instituto Nacional de Câncer. Inquérito domiciliar sobre comportamentos de risco e morbidade referida de doenças e agravos não transmissíveis: Brasil, 15 capitais e Distrito Federal, 2002-2003. Rio de Janeiro: Instituto Nacional de Câncer; 2004.

24. Vargas CM, Ingram DD, Gillum RF. Incidence of hypertension and educational attainment: the NHANES I epidemiologic followup study. First National Health and Nutrition Examination Survey. Am J Epidemiol 2000; 152:272-8.

25. Glover JD, Hetzel DM, Tennant SK. The socioeconomic gradient and chronic illness and associated risk factors in Australia. Aust New Zealand Health Policy 2004; 1:8.

26. Lethbridge-Cejku M, Schiller JS, Bernadel L. Summary health statistics for U.S. adults: National Health Interview Survey, 2002. Vital Health Stat 2004; 222:1-151.

27. Departamento de Informática do SUS. Informações demográficas e socioeconômicas. http://tabnet. datasus.gov.br/tabnet/tabnet.htm\#DemogSocio (acessado em 05/Out/2005).

Recebido em 24/Ago/2007

Versão final reapresentada em 14/Mai/2008 Aprovado em 27/Mai/2008 